\title{
Respiratory muscle training with normocapnic hyperpnea improves ventilatory pattern and thoracoabdominal coordination, and reduces oxygen desaturation during endurance exercise testing in COPD patients
}

This article was published in the following Dove Press journal: International Journal of COPD

10 September 2015

Number of times this article has been viewed

\section{Eva Bernardi' \\ Luca Pomidori' \\ Faisy Bassal' \\ Marco Contoli ${ }^{2}$ \\ Annalisa Cogo'}

'Biomedical Sport Studies Center, University of Ferrara, Ferrara,

${ }^{2}$ Respiratory Section, Department of Medical Sciences, University of Ferrara, Cona (FE), Italy
Correspondence: Eva Bernardi Biomedical Sport Studies Center, University of Ferrara, via Gramicia 35, 44100 Ferrara, Italy

Email bernardi.eva@gmail.com
Background: Few data are available about the effects of respiratory muscle training with normocapnic hyperpnea (NH) in COPD. The aim is to evaluate the effects of 4 weeks of NH (Spirotiger ${ }^{\mathbb{R}}$ ) on ventilatory pattern, exercise capacity, and quality of life (QoL) in COPD patients.

Methods: Twenty-six COPD patients (three females), ages 49-82 years, were included in this study. Spirometry and maximal inspiratory pressure, St George Respiratory Questionnaire, 6-minute walk test, and symptom-limited endurance exercise test (endurance test to the limit of tolerance [tLim]) at $75 \%-80 \%$ of peak work rate up to a Borg Score of 8-9/10 were performed before and after NH. Patients were equipped with ambulatory inductive plethysmography $\left(\right.$ LifeShirt ${ }^{\mathbb{R}}$ ) to evaluate ventilatory pattern and thoracoabdominal coordination (phase angle [PhA]) during tLim. After four supervised sessions, subjects trained at home for 4 weeks 10 minutes twice a day at $50 \%$ of maximal voluntary ventilation. The workload was adjusted during the training period to maintain a Borg Score of 5-6/10.

Results: Twenty subjects completed the study. After NH, maximal inspiratory pressure significantly increased ( $81.5 \pm 31.6$ vs $\left.91.8 \pm 30.6 \mathrm{cmH}_{2} \mathrm{O}, P<0.01\right)$; exercise endurance time ( +150 seconds, $P=0.04), 6$-minute walk test $(+30$ meters, $P=0.03)$, and QoL $(-8, P<0.01)$ all increased. During tLim, the ventilatory pattern changed significantly (lower ventilation, lower respiratory rate, higher tidal volume); oxygen desaturation, $\mathrm{PhA}$, and dyspnea Borg Score were lower for the same work intensity $(P<0.01, P=0.02$, and $P<0.01$, respectively; one-way ANOVA). The improvement in tidal volume and oxygen saturation after $\mathrm{NH}$ were significantly related $\left(R^{2}=0.65, P<0.01\right)$.

Conclusion: As expected, NH improves inspiratory muscle performance, exercise capacity, and QoL. New results are significant change in ventilatory pattern, which improves oxygen saturation and an improvement in thoracoabdominal coordination (lower $\mathrm{PhA}$ ). These two facts could explain the reduced dyspnea during the endurance test. All these results together may play a role in improving exercise capacity after NH training.

Keywords: COPD, exercise, inspiratory muscle, pulmonary rehabilitation

\section{Introduction}

Dyspnea is the primary symptom that limits exercise in many patients with COPD. ${ }^{1,2}$ In COPD, exercise limitation is clearly multifactorial; one of the main contributors ${ }^{1}$ is ventilatory limitation due to increased airway resistance and pulmonary hyperinflation, both increasing the work of breathing. ${ }^{3-5}$ In addition, COPD patients exhibit 
abnormal thoracoabdominal motion during exercise and this is another cause of exercise limitation. ${ }^{6-9}$ In particular, Chien et $\mathrm{al}^{9}$ showed that asynchronous thoracoabdominal motion occurred early during exercise in patients with mild COPD, contributing to a decreased 6-minute walk distance. Respiratory muscle weakness can also play a role in dyspnea perception and exercise limitation. Indeed, inspiratory muscle weakness is observed in COPD patients ${ }^{10,11}$ and contributes to dyspnea perception. ${ }^{1,12,13}$

For this reason, there has been an interest in applying a specific training to the inspiratory muscles in COPD patients. Different forms of respiratory muscle training (RMT) have been used, and from recent meta-analyses ${ }^{14,15}$ it is clear that RMT increases inspiratory muscle performance and functional exercise capacity (ie, 6-minute walk distance, endurance exercise capacity, and maximal oxygen consumption ${ }^{14,15}$ ), and decreases dyspnea, but the clinical importance of these improvements remains unclear. Most studies investigated the effects of RMT with resistive or threshold loading, ${ }^{14}$ while a few studies evaluated ${ }^{16-18}$ the effects of RMT with normocapnic hyperpnea $(\mathrm{NH})$ even though Koppers et al ${ }^{16}$ reported that $\mathrm{NH}$ could be the most appropriate technique for improving respiratory muscle endurance capacity, which is required during endurance exercises.

None of these studies ${ }^{16-18}$ investigated the effects of $\mathrm{NH}$ on thoracoabdominal coordination and oxygen saturation $\left(\mathrm{SpO}_{2}\right)$ during exercise in COPD patients, whereas only one study ${ }^{16}$ investigated the effects of NH on the ventilatory pattern, showing lower minute ventilation and respiratory rate and larger tidal volume during exercise.

The aim of this study was to evaluate the effects of 4 weeks of NH (using the Spirotiger ${ }^{\circledR}$ device) on thoracoabdominal coordination, $\mathrm{SpO}_{2}$, ventilatory pattern, exercise capacity, and quality of life (QoL) in COPD patients.

\section{Methods}

Subjects were consecutively recruited from the outpatient clinic of the Department of Respiratory Disease, Ferrara University Hospital, Italy.

Inclusion criteria were as follows: 1) COPD as defined according to international guidelines, ${ }^{19}$ 2) forced expiratory volume in 1 second $\left(\mathrm{FEV}_{1}\right)$ between $30 \%$ and $80 \%$ predicted, ${ }^{20}$ after bronchodilatation; and 3) stable clinical condition for at least 6 weeks. Exclusion criteria were as follows: 1) cardiac comorbidity (ie, recent myocardial infarction, myocardial ischemia, and cardiac arrhythmia); 2) inability to use the device; and 3) inclusion in any pulmonary rehabilitation program.
The Ethics Committee of the University of Ferrara approved the study (protocol number 090591). Informed consent was obtained from each subject.

\section{Study design}

On the first day of the study, patients performed a respiratory function test (forced flow-volume curve) and their inspiratory muscle strength (maximal inspiratory pressure [MIP]) was measured. After 30 minutes of rest, a 6-minute walk test (6MWT) was performed. ${ }^{21}$ The St George Respiratory Questionnaire (SGRQ) for measuring health-related QoL was completed. ${ }^{22}$

On the second day, an incremental walking test was performed, followed, after 30 minutes of rest, by an endurance test to the limit of tolerance (tLim) (ie, until subjects were unable to continue due to symptoms). Daily physical activity was monitored using the SenseWear Armband ${ }^{\circledR}$ (BodyMedia, Pittsburgh, PA, USA) validated for estimating energy expenditure in COPD patients. ${ }^{23,24}$

After 4 weeks of respiratory muscle endurance training $(\mathrm{NH})$, the subjects repeated all the tests in a standardized manner and sequence.

The testing and the training procedure are detailed in the sections Exercise testing and Respiratory muscle endurance training.

\section{Respiratory function}

The forced flow-volume curve was performed (JAEGER ${ }^{\circledR}$ MasterScreen Body; San Diego, CA, USA) to measure forced vital capacity and $\mathrm{FEV}_{1}$. In accordance with the guidelines of the American Thoracic Society/European Respiratory Society, ${ }^{25}$ subjects repeated the maneuver at least three times and the two largest values of forced vital capacity and FEV $\mathrm{F}_{1}$ had to be within $0.15 \mathrm{~L}$ of each other. Reference normal values were taken from the European Community for Steel and Coal. ${ }^{20}$

After respiratory function, MIP was measured; starting from residual volume, the subject was asked to inspire as deeply as possible, maintaining the maximal pressure for at least 1.5 seconds. The pressure was measured with a manometer (CARDINAL Health microRPM ${ }^{\circledR}$; San Diego, CA, USA). Subjects repeated the maneuver a minimum of five times and reproducibility had to be within 5\%-10\% in accordance with the guidelines. ${ }^{26}$ The highest value was considered for comparison before and after training.

\section{Exercise testing}

The 6MWT was performed in a corridor $24 \mathrm{~m}$ long. The subjects were instructed to cover as much distance as possible 
in 6 minutes and standardized verbal encouragement was given. ${ }^{21}$ The maximum distance walked was recorded.

The maximal incremental exercise test was performed on a treadmill (Runner Galaxy MTC, Cavezzo, Italy). During the test, the work rate was increased every 10 meters by $0.2 \mathrm{~km} / \mathrm{h} .{ }^{27}$ Arterial blood pressure was measured every minute. Heart rate and $\mathrm{SpO}_{2}$ were continuously monitored. At the end of the test, maximal working capacity $\left(\right.$ Speed $_{\max }$ ) was recorded.

A constant-load endurance exercise test was performed on a treadmill at a work rate of $80 \%-85 \%$ of the individual's Speed $_{\text {max }}$ and continued to the tLim. ${ }^{28}$ Subjects were not encouraged during the test. Perception of dyspnea and muscular fatigue were measured using the Borg Scale ${ }^{29}$ at regular intervals. The tLim was terminated when the subjects indicated they were unable to continue due to intolerable dyspnea and/or muscular fatigue (Borg Score 8-9/10), or were unable to keep up with the speed of the treadmill; the duration of the test was recorded as walking endurance time.

During tLim, patients were equipped with ambulatory inductive plethysmography (LifeShirt ${ }^{\circledR}$ System, San Diego, CA, USA). ${ }^{30}$ The LifeShirt ${ }^{\circledR}$ features rib cage and abdominal inductance bands embedded within an elasticized vest, connected to a battery-operated handheld computer. The handheld computer continuously records data. Before each test, a calibration of the system was performed. After the testing session, the data were downloaded and analyzed with a dedicated software (Vivologic 3.0, Vivonoetics, San Diego, CA, USA) for calculating respiratory variables: ventilation, tidal volume, and respiratory rate. The phase angle $(\mathrm{PhA})$ was obtained as a measure of asynchrony between rib cage and abdominal movements (thoracoabdominal asynchrony). A pulse oximeter and an electrocardiograph were integrated into the LifeShirt system.

\section{Respiratory muscle endurance training}

The training protocol lasted 4 weeks and was performed by means of Spirotiger ${ }^{\circledR}$ (MVM, Linate, MI, Italy), which consists of a handheld unit with a pouch and a base station. A two-way piston valve connected to a rebreathing bag permits a constant isocapnic end-tidal $\mathrm{CO}_{2}$ fraction ${ }^{31}$ to be maintained.

Before starting the protocol, subjects underwent four supervised training sessions to learn the technique and to define the target training ventilation. In accordance with previous studies, ${ }^{16-18}$ the size of the bag was adjusted to $50 \%-60 \%$ of the subject's vital capacity and the breathing frequency chosen was such that ventilation corresponded to $50 \%-60 \%$ of maximal voluntary ventilation (calculated as 35 times $\mathrm{FEV}_{1}$ ). The tidal volume was adjusted by changing the size of the bag and breathing frequency was changed accordingly to maintain target training ventilation (ie $50 \%-60 \%$ of maximal voluntary ventilation). The subjects trained 20 minutes daily ( 1 minute of exercise and 1 minute of rest, ten times, twice a day), 7 days a week, for 5 weeks. While performing $\mathrm{NH}$, the patients wore a nose clip to ensure breathing occurred exclusively through the training device.

During the training period, a diary documented the compliance with home-based training: number, duration of the sessions, and rating of perceived respiratory exertion (Borg Score) were reported.

\section{Statistical analysis}

Data are reported as mean \pm standard deviation. The Student's $t$-test was used to compare baseline characteristics and training changes. The one-way ANOVA test with analysis of the covariate was used to evaluate the effects of training on ventilation, ventilatory pattern, and $\mathrm{PhA}$; the $P$-values were adjusted according to the Bonferroni correction. The parametric Pearson correlation coefficient was used to describe the relationships between variables. Statistical significance was accepted at $P<0.05$. All the analyses were performed using GraphPad Prism 40.

\section{Results}

Twenty-six patients were included in the study. Baseline characteristics of the subjects are shown in Table 1. The mean age of the study population was $69 \pm 8$ years. According to Armband $^{\circledR}$ data, the physical activity status of the subjects was between low and moderate (Table 1). Medication was not changed during the study period.

Table I Baseline characteristics (26 subjects)

\begin{tabular}{ll}
\hline Variables & T0 \\
\hline Age (years) & $69 \pm 8$ \\
Weight $(\mathrm{kg})$ & $83.5 \pm 13.2$ \\
Body mass index $\left(\mathrm{kg} / \mathrm{m}^{2}\right)$ & $29.4 \pm 4.8$ \\
$\mathrm{FEV},(\mathrm{L})$ & $1.49 \pm 0.47$ \\
$\mathrm{FEV},(\%$ pred) & $56 \pm 16$ \\
$\mathrm{FVC}(\mathrm{L})$ & $2.63 \pm 0.84$ \\
$\mathrm{FVC}(\%$ pred) & $75 \pm 2 \mathrm{I}$ \\
$\mathrm{FEV} / \mathrm{FVC}(\%)$ & $57 \pm 13$ \\
MIP (cmH $\left.\mathrm{C}_{2} \mathrm{O}\right)$ & $81.5 \pm 31.6$ \\
MIP $(\%$ pred) & $81 \pm 33$ \\
SGRQ (total) & $29.6 \pm 16.8$ \\
Daily physical activity (METs) & $1.4 \pm 0.2$ \\
\hline
\end{tabular}

Note: Data are expressed as mean \pm SD.

Abbreviations: $\mathrm{FEV}_{1}$, forced expiratory volume in I second; $\mathrm{FEV}_{1}$ (\% pred), \% of predicted value of forced expiratory volume in I second; FVC, forced vital capacity; MIP, maximal inspiratory pressure; SGRQ, St George Respiratory Questionnaire; TO, baseline; SD, standard deviation; MET, metabolic equivalent of task. 
Twenty patients completed the study. Six patients did not complete the study: two because of poor tolerance to the device, three because of poor compliance, and one because of excessive fatigue.

Intriguingly, at baseline (T0), a positive correlation is found between MIP and $\mathrm{FEV}_{1}$ as a percentage of predicted values (Figure 1A) and between MIP and the 6MWT (Figure 1B); an inverse correlation is shown between MIP and the total SGRQ score (Figure 1C).

\section{$\mathrm{NH}$ training}

Analysis of the diaries showed that all patients trained 20 minutes daily, every day at a workload corresponding to $51 \%$ of maximal voluntary ventilation with a mean Borg Score of 6/10.

\section{Inspiratory muscle performance}

Table 2 shows the effects of 4 weeks of home-based $\mathrm{NH}$ on respiratory function and muscle strength. After training (T1), MIP significantly increased $\left(+10.3 \mathrm{cmH}_{2} \mathrm{O}\right.$; Student's $t$-test, $P=0.0001)$. A strong correlation was found between the value at T0 and at T1 $\left(R^{2}=0.90, P<0.00001\right)$. The probability plot distribution confirms normal distribution of the data.

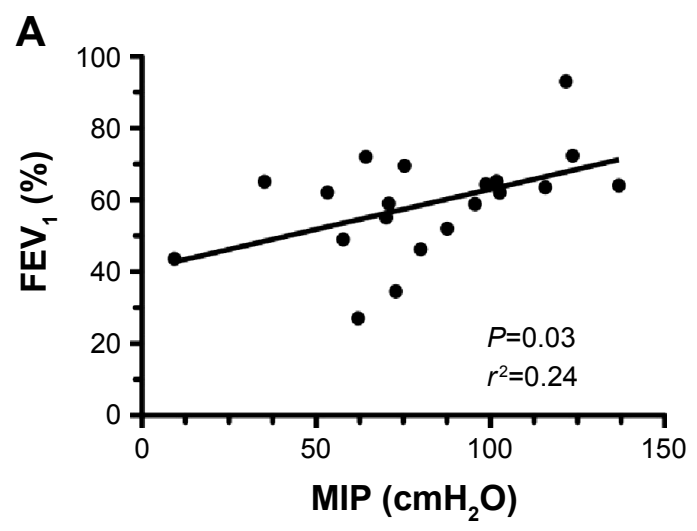

\section{Exercise performance and ventilatory pattern}

The effects of $\mathrm{NH}$ on exercise performance are shown in Table 2.

Regarding the 6MWT, the distance covered increased by $30 \mathrm{~m}$ (Student's $t$-test, $P=0.03$ ).

The duration of thim significantly increased by 2 minutes and 30 seconds (Student's $t$-test, $P=0.04$ ); during the test, average speed was $4.8 \pm 0.9 \mathrm{~km} / \mathrm{h}$, corresponding to $79 \%$ of Speed $_{\max }(6.1 \pm 1.2 \mathrm{~km} / \mathrm{h})$.

Regarding ventilatory data, a significant increase in tidal volume at rest was shown (T0: $0.29 \pm 0.74 \mathrm{~mL}$ and T1: $0.32 \pm 0.81 \mathrm{~mL}$; Student's $t$-test, $P=0.02$ ). During tLim, after the training, significantly lower ventilation and respiratory rate were found (one-way ANOVA, $P<0.05$; Figure $2 \mathrm{~A}-\mathrm{C}$ for ventilation, respiratory rate, and tidal volume, respectively). Furthermore, a lower increase in $\mathrm{PhA}$ was found during exercise (one-way ANOVA, $P=0.02$; Figure 3).

Regarding $\mathrm{SpO}_{2}$, an improvement was found at rest (Student's $t$-test, $P=0.02$; Table 2). The $\mathrm{SpO}_{2}$ monitoring during tLim showed significantly higher values (one-way ANOVA, $P<0.01$; Figure 4).

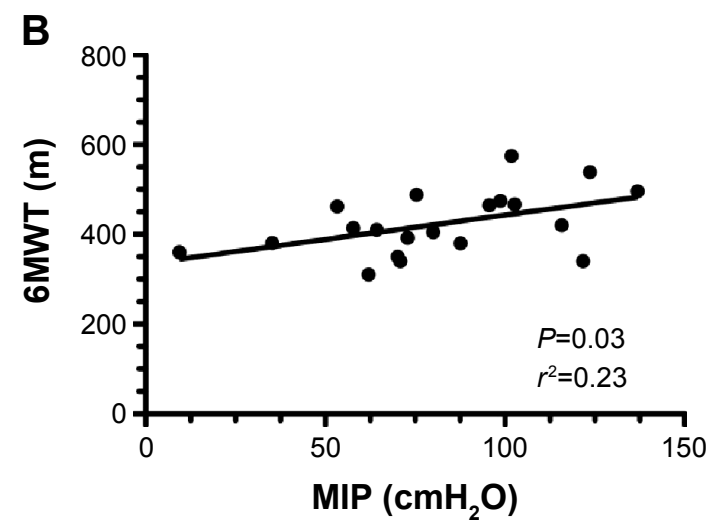

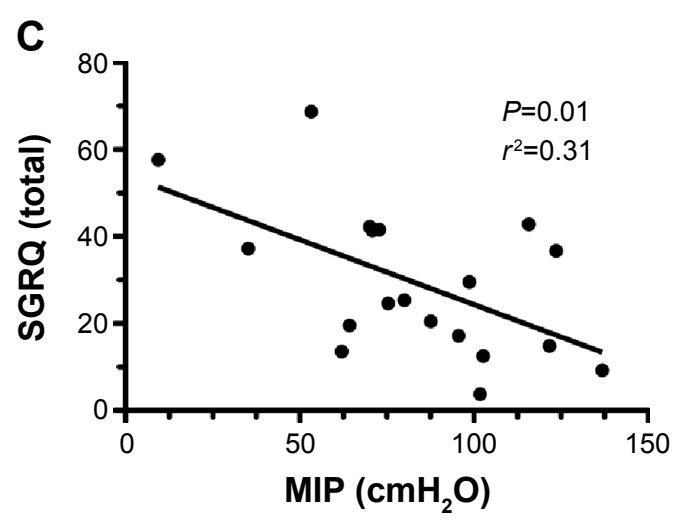

Figure I Correlation between MIP and FEV $(\mathbf{A}), 6 \mathrm{MWT}(\mathbf{B})$, and SGRQ (C) before the training.

Abbreviations: $\mathrm{FEV}_{1}$, forced expiratory volume in I second; 6MWT, 6-minute walk test; SGRQ, St George Respiratory Questionnaire; MIP, maximal inspiratory pressure. 
Table 2 Pretraining and posttraining values (20 subjects)

\begin{tabular}{|c|c|c|}
\hline Variables & T0 & TI \\
\hline \multicolumn{3}{|l|}{ Respiratory function } \\
\hline $\mathrm{FEV}_{1}(\mathrm{~L})$ & $1.54 \pm 0.44$ & $1.56 \pm 0.39$ \\
\hline FVC (L) & $2.57 \pm 0.84$ & $2.82 \pm 0.77$ \\
\hline \multicolumn{3}{|l|}{ Respiratory muscle pressure } \\
\hline $\mathrm{MIP}\left(\mathrm{cmH}_{2} \mathrm{O}\right)$ & $81.5 \pm 31.6$ & $91.8 \pm 30.6 *$ \\
\hline \multicolumn{3}{|l|}{ Exercise performance } \\
\hline 6MWT (meters) & $423 \pm 71$ & $453 \pm 73 *$ \\
\hline \multicolumn{3}{|l|}{ tLim } \\
\hline Time (seconds) & $347 \pm 235$ & $497 \pm 410 *$ \\
\hline Heart rate rest (bpm) & $80 \pm 13$ & $77 \pm 14$ \\
\hline Heart rate $\max (\mathrm{bpm})$ & $123 \pm 19$ & $123 \pm 14$ \\
\hline $\mathrm{SpO}_{2}$ rest (\%) & $94 \pm 2$ & $95 \pm 2 *$ \\
\hline $\mathrm{SpO}_{2} \min (\%)$ & $90 \pm 4$ & $91 \pm 2$ \\
\hline Dyspnea Borg Score (/I0) & $8 \pm 1$ & $8 \pm 1$ \\
\hline Muscular fatigue Borg Score (/I0) & $7 \pm 1$ & $7 \pm 2$ \\
\hline SGRQ (total) & $29 \pm 17$ & $21 \pm 12^{*}$ \\
\hline Daily physical activity (METs) & $1.5 \pm 0.2$ & $1.5 \pm 0.2$ \\
\hline Number of steps & $7,210 \pm 2,388$ & $7,720 \pm 4,199$ \\
\hline
\end{tabular}

Notes: Data are expressed as mean $\pm \mathrm{SD}$. *Student's $t$-test, $P<0.05$.

Abbreviations: $\mathrm{FEV}_{1}$, forced expiratory volume in I second; FVC, forced vital capacity; MIP, maximal inspiratory pressure; 6MWT, 6-minute walk test; thim, endurance test to the limit of tolerance; $\mathrm{SpO}_{2}$, oxygen saturation; SGRQ, St George Respiratory Questionnaire; TO, baseline; TI, after 4 weeks of respiratory muscle training; SD, standard deviation; bpm, beats per minute; MET, metabolic equivalent of task; min, minimum.

After training, a correlation was found during tLim between the changes in tidal volume and $\mathrm{SpO}_{2}$ (Figure 5).

All these findings were associated with lower dyspnea and muscular fatigue Borg Score (one-way ANOVA, $P<0.01$; Figure 6A and B, respectively).

\section{Discussion}

The aim of this study was to evaluate the effects of 4 weeks of inspiratory muscle training with $\mathrm{NH}$ on ventilatory pattern, exercise capacity, and QoL in COPD patients. The main results are the changes in ventilatory pattern and the improved thoracoabdominal coordination during exercise after training.

Regarding the ventilatory pattern, after training at the same work intensity, we found lower ventilation, a decrease in respiratory rate, and an increase in tidal volume. The slower and deeper ventilation allows improved alveolar ventilation, which in turn has an effect on gas exchange. The subjects exhibit improved oxygenation, which is related to the increase in tidal volume. Improved oxygenation is already evident at rest when the ventilatory pattern is characterized by a higher tidal volume at the same ventilation. Several studies ${ }^{32-34}$ have already shown that deeper and slower breathing (so-called yoga breathing) improves $\mathrm{SpO}_{2}$ in both healthy subjects exposed to high altitude ${ }^{32}$ and subjects suffering from chronic diseases such as heart failure ${ }^{33}$ and COPD. ${ }^{34}$

To our knowledge, only Koppers ${ }^{16}$ has described the ventilatory pattern changes after NH, but only during exercise. In his study, ${ }^{16}$ he also highlights that deeper and slower ventilation reduces the work of breathing, delaying respiratory muscle fatigue, and dyspnea perception. Moreover, improved thoracoabdominal coordination plays a key role in dyspnea perception, even though few data are available in literature.
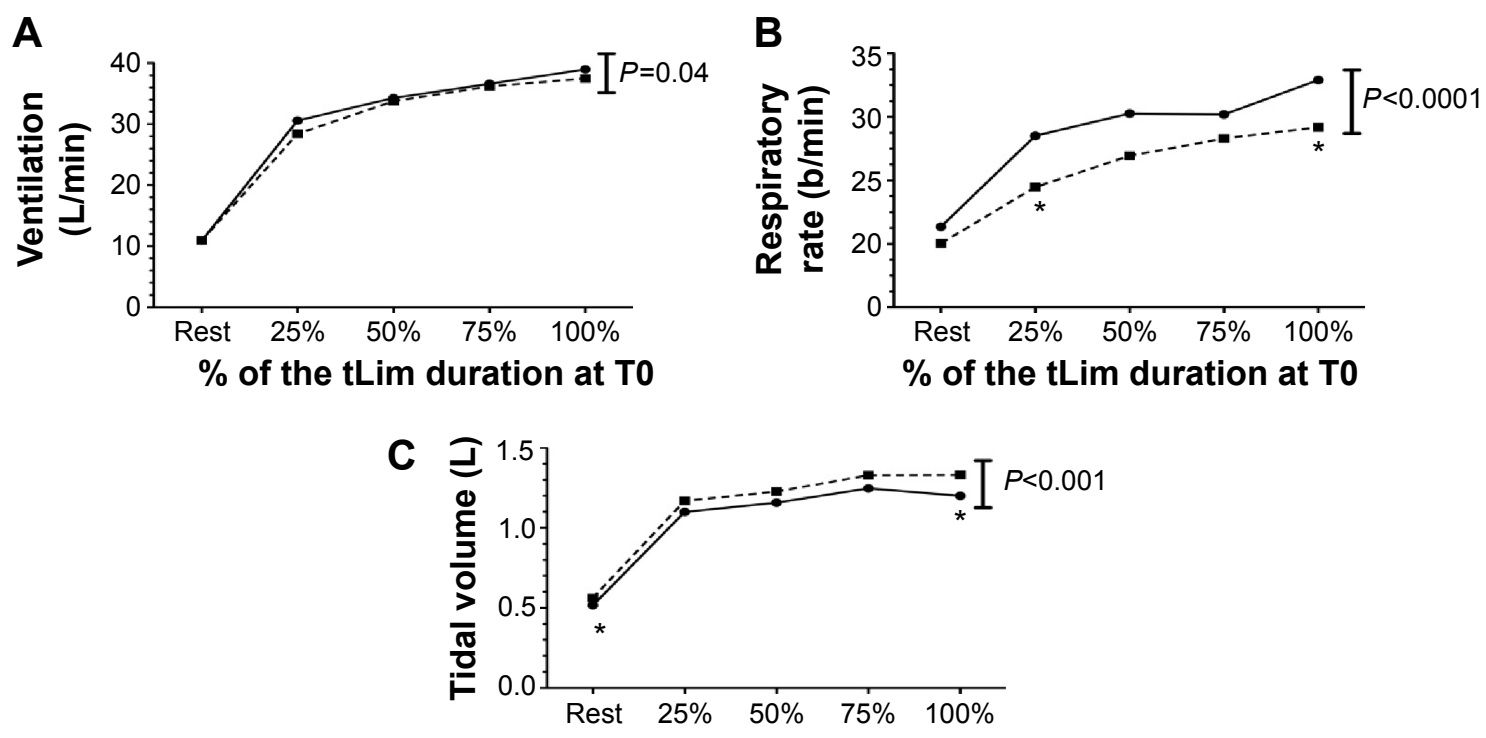

$\%$ of the tLim duration at TO

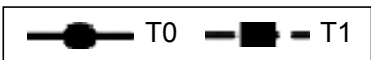

Figure 2 The ventilation $(\mathbf{A})$, respiratory rate $(\mathbf{B})$, and tidal volume trend $(\mathbf{C})$ during tLim before T0 and after $\mathrm{TI}$ training.

Notes: ANOVA test between T0 and TI. Mean value at rest, at $25 \%, 50 \%, 75 \%$, and $100 \%$ of the tLim duration at T0. ${ }^{* P}<0.05$ between T0 and TI.

Abbreviations: tLim, endurance test to the limit of tolerance; TO, baseline; TI, after 4 weeks of respiratory muscle training; ANOVA, analysis of variance; min, minute. 


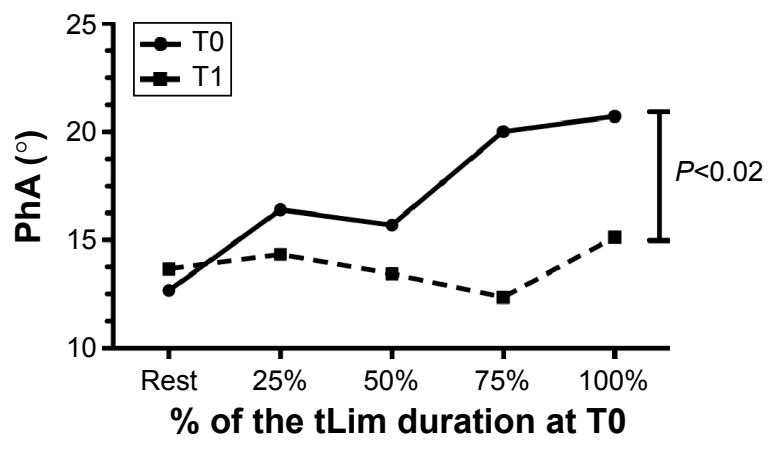

Figure 3 The $\mathrm{PhA}$ trend during thim before and after training. Notes: ANOVA test between T0 and TI. Mean value at rest, at $25 \%, 50 \%, 75 \%$, and $100 \%$ of the thim duration at TO.

Abbreviations: $\mathrm{PhA}$, phase angle; tLim, endurance test to the limit of tolerance; TO, baseline; TI, after 4 weeks of respiratory muscle training.

Indeed, only two studies ${ }^{9,35}$ have investigated this asynchrony in COPD subjects. In particular, $\mathrm{Chien}^{9}$ has shown that thoracoabdominal asynchrony reduces exercise capacity in moderate and severe COPD. The mechanism, however, remains unclear. ${ }^{36,37}$ Our results are in line with this result; in fact, in our study, patients with moderate and severe COPD show an increased exercise capacity together with better thoracoabdominal coordination. Both the change in ventilatory pattern and the improved thoracoabdominal coordination can explain the reduction in dyspnea perception.

Besides the innovative results related to ventilation, we also found a significant increase in inspiratory muscle strength after NH. This is a quite controversial point. In fact, it is clear from meta-analyses ${ }^{14,15}$ that $\mathrm{NH}$ increases inspiratory muscle endurance and only one study ${ }^{18}$ showed that it improved respiratory muscle strength. However, the

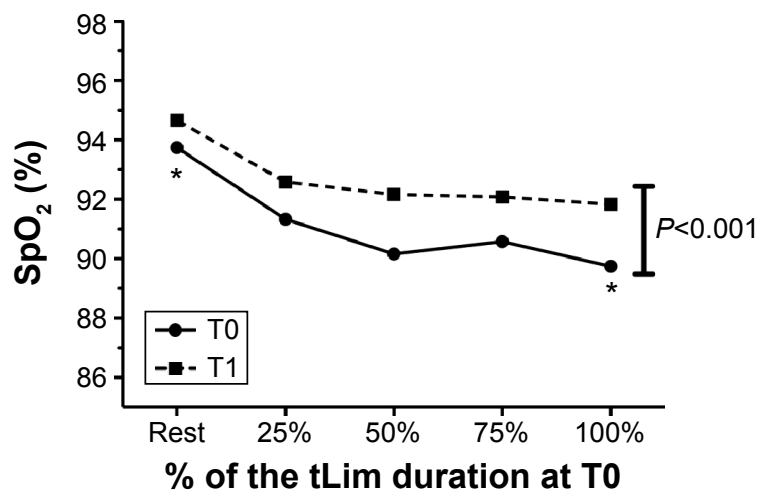

Figure 4 The $\mathrm{SpO}_{2}$ trend during thim before and after training. Notes: ANOVA test between TO and TI. Mean value at rest, at $25 \%, 50 \%, 75 \%$, and $100 \%$ of the tLim duration at T0. $* P<0.05$ between T0 and TI.

Abbreviations: $\mathrm{SpO}_{2}$, oxygen saturation; thim, endurance test to the limit of tolerance; T0, baseline; TI, after 4 weeks of respiratory muscle training; ANOVA, analysis of variance.

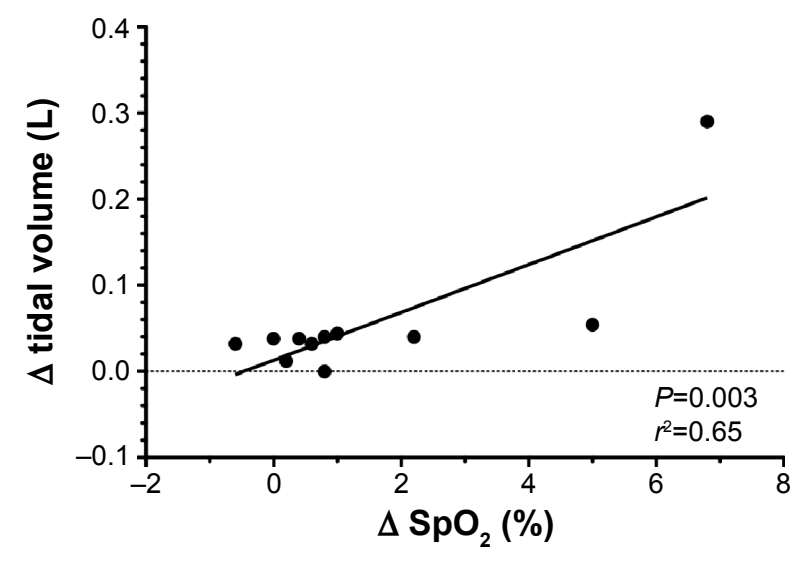

Figure 5 Correlation between the changes in tidal volume and $\mathrm{SpO}_{2}$, before and after training, during thim.

Abbreviations: $\mathrm{SpO}_{2}$, oxygen saturation; thim, endurance test to the limit of tolerance.

increase in respiratory muscle strength, and not just endurance, is clinically relevant to COPD patients. ${ }^{38,39}$ In fact, Terzano et $\mathrm{al}^{38}$ and Tudorache et $\mathrm{al}^{39}$ have demonstrated that airway obstruction is closely associated with lower respiratory muscle pressures. In our study, we also found, at baseline, a correlation between MIP and the severity of bronchial obstruction. Furthermore, Tudorache et $\mathrm{al}^{39}$ found a correlation between MIP and the 6MWT distance, as in our study. However, to the best of our knowledge, there is no study in literature demonstrating a significant inverse correlation between MIP and QoL.

Regarding exercise capacity, the positive effects of $\mathrm{NH}$ in COPD $^{16-18}$ are already known. In this study, we have found a higher duration of the endurance test $(+43 \%)$ and an increase in walking distance during the $6 \mathrm{MWT}(+30 \mathrm{~m})$, which are the minimal important difference. ${ }^{28,40,41}$

Another interesting point is the evidence that the level of physical activity of the subjects did not change during the study period. The use of the multi-sensor Armband ${ }^{\circledR}$ allowed us to verify that the energy expenditure and the number of daily steps did not change significantly after NH. Therefore, no significant physical activity changes could have influenced our results. ${ }^{42}$

This study has some limitations. The main limitation is the absence of a sham group. So far, there is no instrument available that allows NH without any workload, which should be the gold standard for control group training. Among all papers discussing $\mathrm{NH}$, only three ${ }^{17,18,43}$ studied a control group training with an incentive spirometer with minimal resistance and a low respiratory rate; however, this device does not completely satisfy our purposes. 

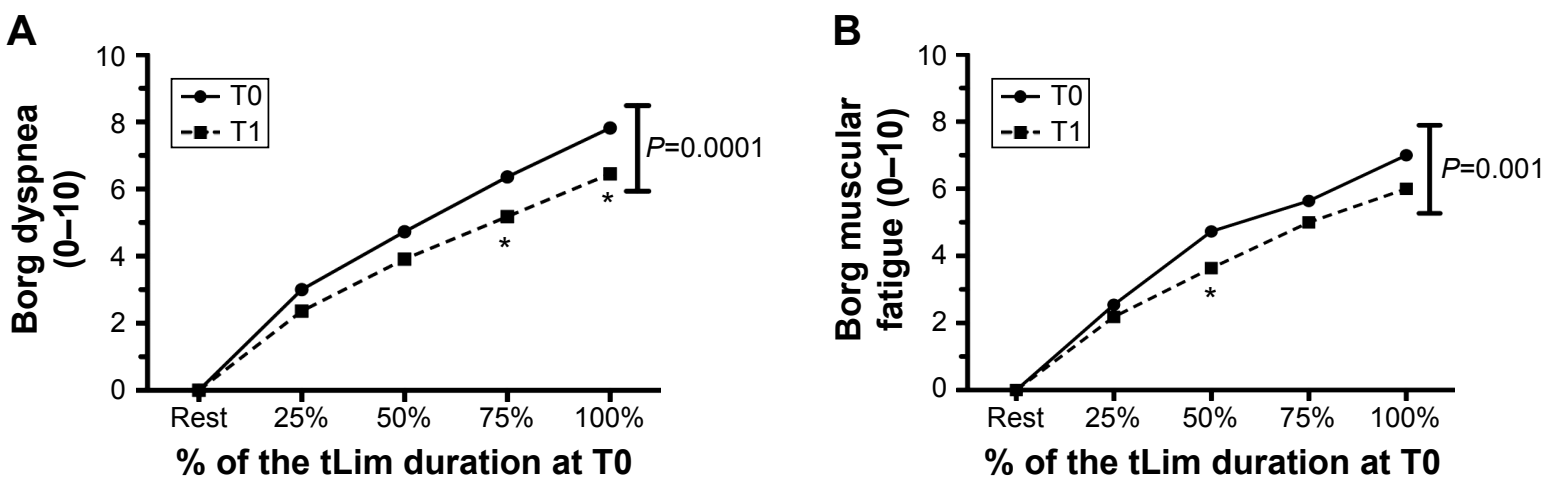

Figure 6 The Borg dyspnea (A) and muscular fatigue (B) trend during tLim before and after training.

Notes: ANOVA test between T0 and TI. Mean value at rest, at $25 \%, 50 \%, 75 \%$, and $100 \%$ of the thim duration at T0. ${ }^{* P}<0.05$ between $\mathrm{T} 0$ and $\mathrm{TI}$.

Abbreviations: tLim, endurance test to the limit of tolerance; TO, baseline; TI, after 4 weeks of respiratory muscle training; ANOVA, analysis of variance.

Another limitation is the high number of dropouts (23\%). A careful analysis of the characteristics of these subjects could have helped us to better identify the subjects that could benefit from this training. However, we did not find any significant differences between the two groups as regards age, respiratory function, exercise capacity, anthropometric characteristics, and QoL. All subjects but one dropped out because of poor compliance and poor adaptation to the device. Therefore, it seems that motivation is the main factor for completing the training.

\section{Conclusion}

$\mathrm{NH}$ is an effective treatment for COPD to improve respiratory muscle strength, ventilatory pattern, and thoracoabdominal coordination, which have a positive effect on $\mathrm{SpO}_{2}$. As a consequence, dyspnea perception decreases, exercise capacity increases, and QoL improves. These preliminary results seem to show that $\mathrm{NH}$ can be used as a nonpharmacological therapy to complement exercise, or as an alternative aid for COPD patients to improve exercise capacity and QoL.

\section{Acknowledgment}

The text was revised by a professional native English person (Help S.r.1., Ferrara, Italy).

\section{Author contributions}

All authors contributed toward data analysis, drafting and revising the paper and agree to be accountable for all aspects of the work.

\section{Disclosure}

This study had no financial support. The authors report no conflicts of interest in this work.

\section{References}

1. O'Donnel DE, Laveneziana P. Dyspnea and activity limitation in COPD: mechanical factors. COPD. 2008;4:225-236.

2. Gosselink R, Troosters T, Decramer M. Peripheral muscle weakness contributes to exercise limitation in COPD. Am J Respir Crit Care Med. 1996;153:976-980.

3. Nici L. Mechanisms and measures of exercise intolerance in chronic obstructive pulmonary disease. Clin Chest Med. 2000;21:693-704.

4. Sinderby C, Spahija J, Beck J, et al. Diaphragm activation during exercise in chronic obstructive pulmonary disease. Am J Respir Crit Care Med. 2001;163:1637-1641.

5. O'Donnell DE, Bertley JC, Chau LK, Webb KA. Qualitative aspects of exertional breathlessness in chronic airflow limitation: pathophysiologic mechanisms. Am J Respir Crit Care Med. 1997;155:109-115.

6. Alves GS, Britto RR, Campos FC, Vilaca AB, Moraes KS, Parreira VF. Breathing pattern and thoracoabdominal motion during exercise in chronic obstructive pulmonary disease. Braz J Med Biol Res. 2008;41(11):945-950.

7. Sharp JT, Goldberg NB, Druz WS, Fishman HC, Danon J. Thoracoabdominal motion in chronic obstructive pulmonary disease. Am Rev Respir Dis. 1977;115(1):47-56.

8. Delgado HR, Braun SR, Skatrud JB, Reddan WG, Pegelow DF. Chest wall and abdominal motion during exercise in patients with chronic obstructive pulmonary disease. Am Rev Respir Dis. 1982;126(2): 200-205.

9. Chien JY, Ruan SY, Huang YC, Yu CJ, Yang PC. Asynchronous thoraco-abdominal motion contributes to decreased 6-minute walk test in patients with COPD. Respir Care. 2013;58(2):320-326.

10. Decramer M, Demedts M, Rochette F, Billet L. Maximal transrespiratory pressures in obstructive lung disease. Bull Eur Physiopathol Respir. 1980;16:479-490.

11. Polkey MI, Kyroussis D, Hamnegard CH, Mills GH, Green M, Moxham J. Diaphragm strength in chronic obstructive pulmonary disease. Am J Respir Crit Care Med. 1996;154:1310-1317.

12. Killian KJ, Jones NL. Respiratory muscles and dyspnea. Clin Chest Med. 1988;9:237-248.

13. Hamilton N, Killian KJ, Summers E, Jones NL. Muscle strength, symptom intensity, and exercise capacity in patients with cardiorespiratory disorders. Am J Respir Crit Care Med. 1995;152:2021-2031.

14. Gosselink R, De Vos J, Van Den Heuvel SP, Segers J, Decramer M, Kwakkel G. Impact of inspiratory muscle training in patients with COPD: what is the evidence? Eur Respir J. 2011;37(2):416-425.

15. Geddes EL, O’Brien K, Reid WD, Brooks D, Crowe J. Inspiratory muscle training in adults with chronic obstructive pulmonary disease: an update of a systematic review. Respir Med. 2008;102(12): $1715-1729$. 
16. Koppers RJ, Vos PJ, Boot CR, Folgering HT. Exercise performance improves in patients with COPD due to respiratory muscle endurance training. Chest. 2006;129(4):886-892.

17. Scherer TA, Spengler CM, Owassapian D, Imhof E, Boutellier U. Respiratory muscle endurance training in chronic obstructive pulmonary disease: impact on exercise capacity, dyspnea, and quality of life. Am J Respir Crit Care Med. 2000;162(5):1709-1714.

18. Mador MJ, Deniz O, Aggarwal A, Shaffer M, Kufel TJ, Spengler CM. Effect of respiratory muscle endurance training in patients with COPD undergoing pulmonary rehabilitation. Chest. 2005;128(3): 1216-1224

19. Global Strategy for the Diagnosis, Management, and Prevention of COPD Global Initiative for Chronic Obstructive Pulmonary disease $(G O L D) ; 2011$. Available from: www.goldcopd.com. Accessed May 12, 2015.

20. European Community for Steel and Coal. Standardized lung function testing. Eur Respir J. 1993;16:25-27.

21. ATS Committee on Proficiency Standards for Clinical Pulmonary Function Laboratories. ATS statement: guidelines for the six-minute walk test. Am J Respir Crit Care Med. 2002;166:111-117.

22. Jones PW, Quirk FH, Baveystock CM. The St George's respiratory questionnaire. Respir Med. 1991;85(suppl B):25-31.

23. Patel SA, Benzo RP, Slivka WA, Sciurba FC. Activity monitoring and energy expenditure in COPD patients: a validation study. COPD. 2007;4:107-112.

24. Van Remoortel H, Raste Y, Louvaris Z, et al; PROactive consortium. Validaty of six activity monitors in chronic obstructive disease: a comparison with indirect calorimetry. PLoS One. 2012;7(6):e39198.

25. Miller MR, Hankinson J, Brusasco V, et al. Standardization of spirometry. Eur Respir J. 2005;26:319-338.

26. American Thoracic Society/European Respiratory Society. ATS/ERS Statement on respiratory muscle testing. Am J Respir Crit Care Med. 2002;166(4):518-624.

27. Manfredini F, Conconi F, Malagoni AM, et al. Speed rather than distance: a novel graded treadmill test to assess claudication. Eur J Vasc Endovasc Surg. 2004;28:303-309.

28. Puente-Maestu L, Villar F, de Miguel J, et al. Clinical relevance of constant power exercise duration changes in COPD. Eur Respir J. 2009; 34(2):340-345.

29. Borg GA. Psycho-physical bases of perceived exertion. Med Sci Sports Exerc. 1982;14:377-381.

30. Clarenbach CF, Senn O, Brack T, Kohler M, Bloch KE. Monitoring of ventilation during exercise by a portable respiratory inductive plethysmography. Chest. 2005;128:1282-1290.

31. Verges S, Boutellier U, Spengler CM. Effect of respiratory muscle endurance training on respiratory sensations, respiratory control and exercise performance: a 15-year experience. Respir Physiol Neurobiol. 2008;161:16-22.
32. Bernardi L, Passino C, Wilmerding V, et al. Breathing patterns and cardiovascular autonomic modulation during hypoxia induced by simulated altitude. J Hypertens. 2001;19(5):947-958.

33. Bernardi L, Spadacini G, Bellwon J, Hajiric R, Roskamm H, Frey AW. Effect of breathing rate on oxygen saturation and exercise performance in chronic heart failure. Lancet. 1998;35:1308-1311.

34. Pomidori L, Campigotto F, Amatya TM, Bernardi L, Cogo A. Efficacy and tolerability of yoga breathing in patients with chronic obstructive pulmonary disease: a pilot study. J Cardiopulm Rehabil Prev. 2009;29: 133-137.

35. Priori R, Aliverti A, Albuquerque AL, Quaranta M, Albert P, Calverley PMJ. The effect of posture on asynchronous chest wall movement in COPD. Appl Physiol. 2013;114(8):1066-1075.

36. Sharp JT, Drutz WS, Moisan T, Foster J, Machnach W. Postural relief of dyspnea in severe chronic obstructive pulmonary disease. Am Rev Respir Dis. 1980;122(2):201-211.

37. Gosselink RA, Wagenaar RC, Rijswijk H, Sargeant AJ, Decramer ML. Diaphragmatic breathing reduces efficiency of breathing in patients with chronic obstructive pulmonary disease. Am J Respir Crit Care Med. 1995;151(4):1136-1142.

38. Terzano C, Ceccarelli D, Conti V, Graziani E, Ricci A, Petroianni A. Maximal respiratory static pressures in patients with different stages of COPD severity. Respir Res. 2008;21(9):8.

39. Tudorache V, Oancea C, Mlădinescu OF. Clinical relevance of maximal inspiratory pressure: determination in COPD exacerbation. Int J Chron Obstruct Pulmon Dis. 2010;5:119-123.

40. Holland AE, Hill CJ, Rasekaba T, Lee A, Naughton MT, McDonald CF. Updating the minimall important difference for six-minute walk distance in patients with chronic obstructive pulmonary disease. Arch Phys Med Rehabil. 2010;91:221-225.

41. Polkey MI, Spruit MA, Edwards LD, et al. Six minute-walk test in chronic obstructive pulmonary disease: minimal critically important difference for death or hospitalization. Am J Respir Crit Care Med. 2013; 187(4):82-86

42. Trooster T, Gosselink R, Decramer M. Short- and long- effects of pulmonary rehabilitation in patients with chronic obstructive pulmonary disease: a randomized trial. Am J Med. 2000;109(3):207-212.

43. Verges S, Lenherr O, Haner AC, Schulz C, Spengler CM. Increased fatigue resistance of respiratory muscles during exercise after respiratory muscle endurance training. Am J Physiol Regul Integr Comp Physiol. 2007;292:R1246-R1253.
International Journal of COPD

\section{Publish your work in this journal}

The International Journal of COPD is an international, peer-reviewed journal of therapeutics and pharmacology focusing on concise rapid reporting of clinical studies and reviews in COPD. Special focus is given to the pathophysiological processes underlying the disease, intervention programs, patient focused education, and self management protocols.

\section{Dovepress}

This journal is indexed on PubMed Central, MedLine and CAS. The manuscript management system is completely online and includes a very quick and fair peer-review system, which is all easy to use. Visit http://www.dovepress.com/testimonials.php to read real quotes from published authors. 